külhöhle und besseren Zugänglichkeit sollte der aktivierte Komplex in (2) leichter zu erreichen sein als in (1) - müßte bei der durch (2) katalysierten Umsetzung größer sein als bei der durch (1) katalysierten. In der Tat liegt die freie Aktivierungsenergie der Spontanreaktion zwischen der der schnelleren - Zusatz von (2) - und der der langsameren Katalysereaktion - Zusatz von (1).

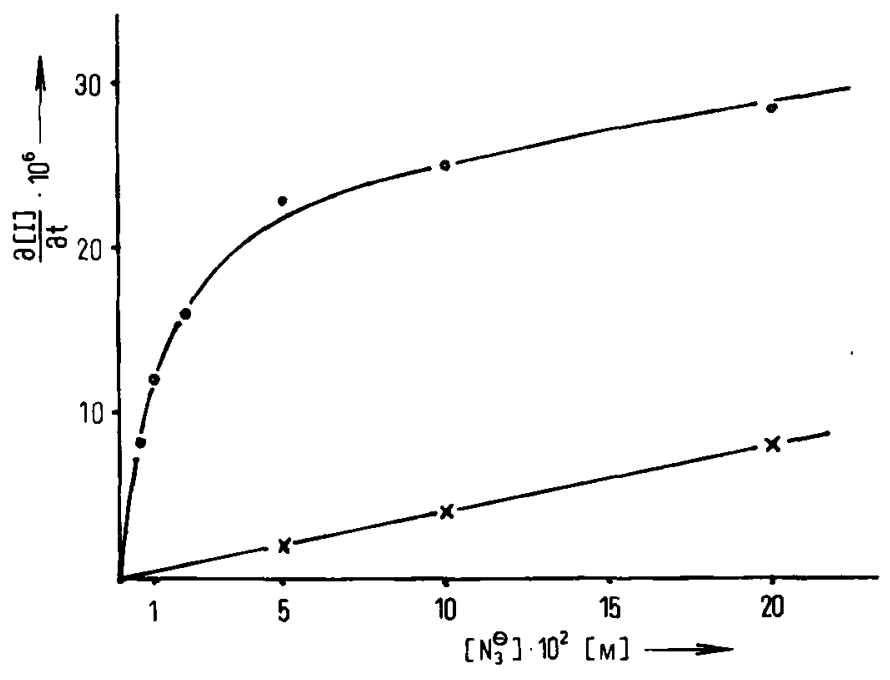

Abb. 1. Kinetik (Initialgeschwindigkeiten $\mathrm{mol} / \mathrm{dm}^{3} \cdot \mathrm{min}$ ) der Reaktion (b) in 25proz. Methanol bei $27^{\circ} \mathrm{C}$ ohne $(x)$ und mit (o) (2) $\left(10^{-2} \mathrm{M}\right)$; $\left[\mathrm{CH}_{3} \mathrm{I}\right]=10^{\cdots 2} \mathrm{M}$

Die Reaktionsbeschleunigung enzymkatalysierter Substitutionen rührt zu einem beträchtlichen Teil vom Wechsel im Mechanismus her: die Spontanreaktion verläuft bimolekular, die enzymkatalysierte Umsetzung unimolekular ${ }^{[1 !}$. Als Beispiel, bei dem der entropische Effekt nicht zum Tragen kommt, untersuchten wir den unimolekularen Zerfall von erythro-2,3-Dibrom-3-phenylpropionat (DBPP) [Gl. (c) $]^{[5]}$. In wäßrigem Methanol zerfällt DBPP auf zwei Wegen $(A$ und $B$ ) zu den isomeren $\beta$-Bromstyrolen: Während die synchrone Fragmentierung ausschließlich das $Z$-Isomer ergibt, wird durch unimolekulare Dissoziation an der Benzylposition ein Carbeniumion erzeugt, das durch Abspaltung von $\mathrm{CO}_{2}$ in die Produkte im thermodynamischen $E / Z$-Verhältnis $(E / Z=9 / 1)$ übergeht.

In der Spontanreaktion ( $\left.[\mathrm{DBPP}]=2 \cdot 10^{-2} \mathrm{M} ; T=27^{\circ} \mathrm{C}\right)$ erhielten wir nach 200 min $20 \%$ Umsatz und ein $E / Z$-Verhältnis der Bromstyrole von 1.64. Die gleiche Reaktion ergab bei Zusatz von $4 \cdot 10^{-2} \mathrm{~mol}$ (2) nach 200 min $36 \%$ Umsatz und ein $E / Z$-Verhältnis 0.48 . Dies zeigt, daß (2) vornehmlich - wenn nicht ausschließlich - die Bildung des Z-Bromstyrols katalysiert. Hier wird noch eine weitere enzymanaloge Eigenschaft des Wirtmoleküls (2) deutlich: Von den beiden Reaktionsweisen des Substrates wird die spezifisch beschleunigt, die über den leichter polarisierbaren anionischen Übergangszustand verläuft. Reaktion (c) beweist, daß die Geschwindigkeitssteigerungen bei der Katalyse durch (2) nicht allein der Änderung der Molekularität der Reaktion (proximity effect, Reaktionen (a) und (b)) zugeschrieben werden können.

Die Geschwindigkeitsänderungen (bei den Reaktionen (a), (b) und (c)) können qualitativ richtig mit den Hughes-Ingold-Regeln vorausgesagt werden, wenn man (1) und (2) als dipolar aprotonische „,Solventien“ ansieht. Die Reaktionsbeschleunigung von Anionenreaktionen durch (2) beim Übergang vom protonischen in ein aprotonisches Medium hat jedoch nicht in der Destabilisierung des Grundzustandes ${ }^{[6]}$, sondern in der Stabilisierung des Übergangszustandes ihre Ursache.
Das synthetische, makrotricyclische Ammoniumsalz (2) nimmt Substrate in eine Molekülhöhle auf und beschleunigt Reaktionen im Einschlußkomplex durch Erniedrigung der Energie des Übergangszustandes. Es weist eine Sättigungskinetik auf und zeigt Substratspezifität sowie Reaktionsspezifität, wegen der hochsymmetrischen Molekülarchitektur allerdings keine Stereospezifität. Aufgrund dieser weitgehenden Analogie $\mathrm{zu}$ den Biokatalysatoren schlagen wir für (2) die Bezeichnung „künstliches Enzym" vor.

Eingegangen am 24. Oktober 1980 [Z 748]

[1] A. R. Fersht: Enzyme Structure and Mechanism, Freeman, San Francisco 1977

[2] J. H. Fendler, E. J. Fendler: Catalysis in Micellar and Macromolecular Systems, Academic Press, London 1975; J. R. Knowles, J. Boger, J. Am. Chem. Soc. 101, 7631 (1979); R. Breslow, M. F. Czarniecki, J. Emert, H. Hamaguchi ibid. 102, 762 (1980); I. Tabushi, Y. Kuroda, A. Mochizuki, ibid. 102, 1152 (1980).

[3] F. P. Schmidtchen, Angew. Chem. 89, 751 (1977); Angew, Chem. Int. Ed. Engl. 16, 720 (1977); Chem. Ber. 1/3, 864 (1980).

[4] F. P. Schmidichen, Chem. Ber. 114, 597 (1981).

[5] E. Grovenstein Jr., D. E. Lee, J. Am. Chem. Soc. 75, 2639 (1953).

[6] A. J. Parker, Chem. Rev. 69, 1 (1969).

\section{Chemilumineszenz von}

Tricarbonyl(chloro)(1,10-phenanthrolin)rhenium(1)

während der katalytischen Zersetzung von Tetralinylhydroperoxid ${ }^{\left.*^{*+}\right]}$

Von Arnd Vogler und Horst Kunkely ${ }^{[*]}$

Die thermische Zersetzung organischer Peroxide ${ }^{[1 \mathrm{a}]}$ oder von Wasserstoffperoxid ${ }^{[16]}$ wird durch Übergangsmetallkomplexe katalysiert. Auch in biologischen Systemen ist diese Reaktion von großer Bedeutung. In den letzten Jahren konnten insbesondere Schuster et al. zeigen, daß selbst organische Verbindungen imstande sind, die Peroxidzersetzung zu katalysieren ${ }^{[2]}$. Die bei dieser Zersetzung freiwerdende Energie reicht aus, um den Katalysator in einen elektronisch angeregten Zustand zu versetzen; die Folge ist eine Chemilumineszenz. Dabei muß der Katalysator im ersten Schritt durch das Peroxid oxidiert werden. Die elektronische Anregung geschieht im letzten Schritt durch die Reduktion des oxidierten Katalysators. Es lag nun nahe zu prüfen, ob dieser Mechanismus auch für die Katalyse durch Übergangsmetallkomplexe gilt.

$\left[\operatorname{Re}(\right.$ phen $\left.)(\mathrm{CO})_{3} \mathrm{Cl}\right] \quad(1)$

schien uns für solche Untersuchungen geeignet, da dieser Komplex sowohl elektrochemisch reversibel oxidiert werden kann $(+1.3 \mathrm{~V}$ vs. SCE) als auch eine elektrochemische Lumineszenz aufweist ${ }^{[3]}$. Außerdem sollte versucht werden, unsere Kenntnisse über die Chemilumineszenz von Übergangsmetallkomplexen, über die nur sehr wenig bekannt ist ${ }^{[4]}$, zu erweitern.

Während Tetralinylhydroperoxid (2) in siedendem Tetralin sich nur langsam zersetzt, erfolgt nach Zugabe des Komplexes (1) eine helle Lichtemission unter gleichzeitigem raschen Zerfall von (2) in $\alpha$-Tetralon (3) und Wasser ${ }^{[5]}$.

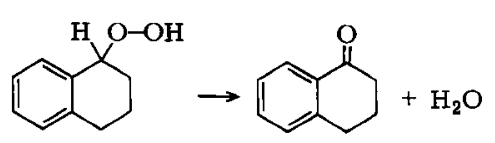

(2)

(3)

[*] Prof. Dr. A. Vogler, Dr. H. Kunkely Institut für Anorganische Chemie der Universität Postfach, D-8400 Regensburg

[**] Diese Arbeit wurde von der Deutschen Forschungsgemeinschaft und dem Fonds der Chemischen Industrie unterstützt. 
Das Spektrum dieser Chemilumineszenz (Abb. 1) ist identisch mit dem der Photolumineszenz von reinem (1) ${ }^{[6]}$. Wie aus der linearen Abhängigkeit der reziproken Chemilumineszenzintensität von der reziproken Komplexkonzentration

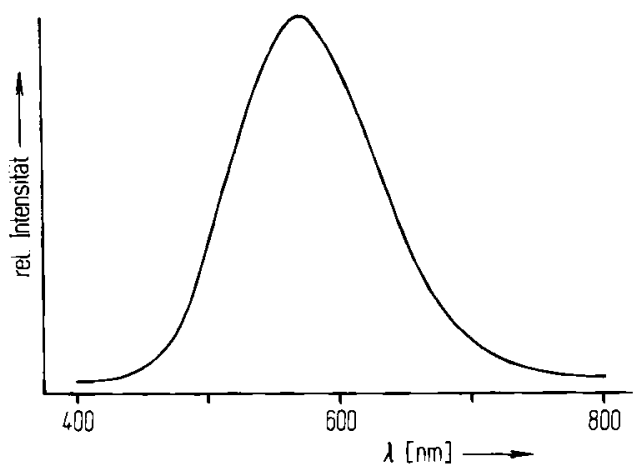

Abb. 1. Chemilumineszenzspektrum der durch $\left[\operatorname{Re}(\right.$ phen $\left.)(\mathrm{CO})_{3} \mathrm{Cl}\right](t)$ katalysierten Zersetzung von Tetalinylhydroperoxid (2).

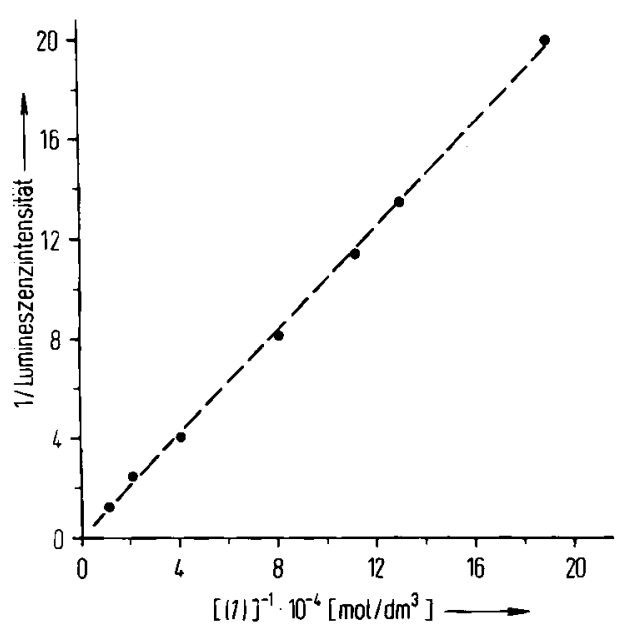

Abb. 2. Abhängigkeit der reziproken Chemilumineszenzintensität (in relativen Einheiten) von der reziproken Konzentration von (1)

hervorgeht ${ }^{[7]}$ (Abb. 2), wird der elektronisch angeregte Rheniumkomplex unmittelbar als Folge einer bimolekularen $\mathrm{Re}-$ aktion gebildet. Danach sollte die Katalyse nach dem Schuster-Mechanismus verlaufen:

$(1)+(2) \rightarrow(1)^{\oplus}(2)^{\ominus}$

$(1)^{\oplus}(2)^{\ominus} \rightarrow(1)^{\oplus}(3)^{\ominus}+\mathrm{H}_{2} \mathrm{O}$

$(1)^{\oplus}(3)^{\ominus} \rightarrow(1)^{*}+(3)$

$(1)^{*} \quad \rightarrow(1)+$ Licht

Obwohl weitere Untersuchungen zur Bestätigung dieses Mechanismus durchgeführt werden müssen ${ }^{[8]}$, soll schon jetzt darauf hingewiesen werden, daß unsere Beobachtung möglicherweise von grundsätzlicher Bedeutung für das Verständnis der katalytischen Peroxidzersetzung durch Übergangsmetallkomplexe ist. Viele Komplexe erfüllen die Voraussetzung, daß sie reversibel oxidiert werden können und energetisch niedrig liegende Anregungszustände haben, die die Zersetzungsenergie der Peroxide aufnehmen können. Das Auftreten einer Lumineszenz ist dabei nicht erforderlich. Unter geeigneten Umständen könnte eine Umkehrung dieser Hydroperoxidzersetzung, d. h. die photochemisch katalysierte Anlagerung von Wasser an ein Keton, erreicht werden ${ }^{[b]}$; das wäre eine Möglichkeit der chemischen Speicherung von Lichtenergie.
[1] a) Ubersicht: D. Swern: Organic Peroxides, Vol. I-III. Wiley-Interscience, New York 1969, 1971 bzw. 1972; b) A. C. Melnyk, N. K. Kildahl, A. R. Rendina, D. H. Busch, J. Am. Chem. Soc. 101, 3232 (1979); H. Sigel, K. Wyss, B. E. Fischer, B. Prijs. Inorg. Chem. 18, 1354 (1979), zit. Lit.

[2] Übersicht: G. B. Schuster, Acc. Chem. Res. 12, 366 (1979).

[3] J. C. Luong, L. Nadjo, M. S. Wrighton, J. Am. Chem. Soc. 100, 5790 (1978).

[4] a) F. E. Lyttle, D. M. Hercules, Photochem. Photobiol. 13, 123 (1971); H. D. Gafney, A.W. Adamson, J. Chem. Educ. 52, 480 (1975); b) A. Vogler, L. ElSayed, R. G. Jones, J. Namnath, A. W. Adamson, Inorg. Chim. Acta 53, L 35 (1981).

[5] Der Zerfall von (2), katalysiert durch $\mathrm{Mg}$ - und $\mathrm{Zn}$-Porphyrine, die dabei eine Chemilumineszenz aufweisen, ist schon lange bekannt: a) $J$. $H$. Helberger, $D$. B. Hever, Ber. Dtsch. Chem. Ges. 72, 11 (1939); b) H. Linschitz in McElroy, Glass: Light and Life, John Hopkins Press, Baltimore 1961, S. 173.

[6] M. Wrighton, D. L. Morse, J. Am. Chem. Soc. 96, 998 (1974).

[7] J.-Y. Koo, G. B. Schuster, J. Am. Chem. Soc. 99, 6107 (1977); 100, 4496 (1978).

[8] Auch $\left[\mathrm{Ru}(\mathrm{bpy})_{3}\right]^{2+}$ chemiluminesziert, wenn auch nur schwach, bei der katalysierten Zersetzung von (2) in Tetralin/Dimethylsulfoxid.

\section{Ein Tripeldecker-Sandwich-Komplex des Rhodiums mit 1,4-Diborabenzol als Brückenligand ${ }^{[* *]}$}

Von Gerhard E. Herberich, Bernd Heßner, Gottfried Huttner und Laszlo Zsolnai $\left.{ }^{*}\right]$

Die bekannten Tripeldecker-Komplexe ${ }^{(1)}$ haben zumeist 7-Ecken-closo-Strukturen mit der pentagonalen Bipyramide als Grundgerüst. Als zentraler Ligand fungiert ein fünfgliedriger Ring, und die apicalen Positionen sind mit $\mathrm{Mn}, \mathrm{Fe}, \mathrm{Co}$ oder Ni besetzt. Die Mehrzahl dieser Komplexe enthält 30 Valenzelektronen (gezählt nach dem Edelgasformalismus) und gehorcht damit den Clusterregeln ${ }^{[2]}$. Komplexe mit 29 und 31-34 Valenzelektronen sind bekannt ${ }^{[3]}$; in ihnen liegt kein anderer Strukturtyp vor, sondern es tritt nur eine Schwächung der Clusterbindungen auf ${ }^{[4]}$

Der vom hypothetischen 1,4-Dimethyl-1,4-dibora-2,5-cyclohexadien (1) abgeleitete Sandwich-Komplex $(2)^{[5]}$ bildet in $\mathrm{CF}_{3} \mathrm{COOH}$ das neuartige, blaßgelbe Tripeldecker-Sandwichkation $(3)^{2+}$. Das gleiche Kation wird auch aus (2) durch Aufstockung mit $\left[\left(\mathrm{C}_{5} \mathrm{Me}_{5}\right) \mathrm{RhCl}_{2} \mathrm{l}_{2} / \mathrm{AlCl}_{3}\right.$ in $\mathrm{CH}_{2} \mathrm{Cl}_{2}$ erhalten.

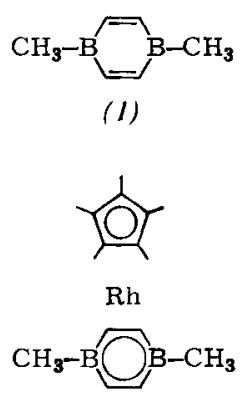

(2)

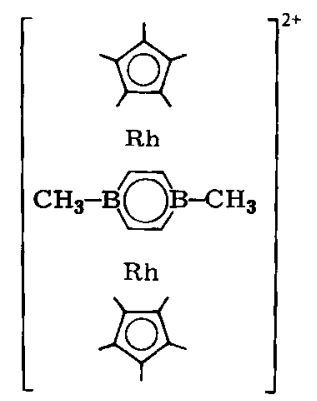

$(3)^{2+}$
[*] Prof. Dr. G. E. Herberich, Dr. B. Heßner Institut für Anorganische Chemie der Technischen Hochschule Professor-Pirlet-Straße 1, D-5100 Aachen

Prof. Dr. G. Huttner, Dipl.-Chem. L. Zsolnaj Fachbereich Chemie der Universität D-7750 Konstanz

[**] Tripeldecker-Komplexe, 2. Mitteilung. Diese Arbeit wurde von der Deutschen Forschungsgemeinschaft und vom Fonds der Chemischen Industrie unterstützt. - 1. Mitteilung: G. E. Herberich, J. Hengesbach, U. Kölle, G. Huttner, A. Frank, Angew. Chem. 88, 450 (1976); Angew. Chem. Int. Ed. Engl. 15, 433 (1976) 